\title{
Substance use and decision-making in adolescent best friendship dyads: The role of popularity
}

\author{
Erik de Water | William J. Burk | Antonius H. N. Cillessen | \\ Anouk Scheres
}

Behavioural Science Institute, Radboud University, Nijmegen, The Netherlands

\section{Correspondence}

Erik de Water, Behavioural Science Institute, Radboud University, Montessorilaan 3, PO Box 9104, 6500 HE Nijmegen,

The Netherlands.

Email: erikdewater@gmail.com

\begin{abstract}
In adolescent best friendship dyads, we examined: (a) similarity in substance use and decision-making; (b) associations between participants' decision-making and their own and best friend's substance use, (c) the influence of relative popularity within the dyad on these associations. Participants ( $n=172 ; 12-18$ years) named their best friend, completed popularity ratings, and a substance use questionnaire. Computer tasks were administered to assess risk-taking and immediate reward preferences. Reciprocated same-sex best friendship dyads ( $n=49$ ) were distinguished on their popularity, and we controlled for age differences between dyads in the analyses. Best friends were similar in substance use and risk-taking preferences. More popular friends' risk-taking preferences were positively associated with alcohol use of less popular friends. These findings underscore best friendship similarity in risky behaviors, and the influence of popular friends.
\end{abstract}

\section{KEYWORDS}

adolescence, alcohol use, decision-making, friendship, sociometric status

\section{1 | INTRODUCTION}

Adolescents engage in heightened levels of risky behaviors, such as substance use, relative to children and adults (Steinberg, 2008). The peer group becomes highly important during adolescence (Steinberg \& Morris, 2001), and adolescents prioritize being popular with their peers over many other goals in life (LaFontana \& Cillessen, 2010). Therefore, it is not surprising that peers strongly influence adolescents' risky behavior (Gardner \& Steinberg, 2005).

Best friends are particularly potent sources of peer influence. Adolescent best friends are highly similar in their alcohol, tobacco, and marijuana use (Burk, van der Vorst, Kerr, \& Stattin, 2012; Kiuru, Burk, Laursen, Salmela-Aro, \& Nurmi, 2010). This similarity is due to selection processes (choosing friends who are similar to oneself) and socialization processes (adopting the behaviors of one's best friend over time) (Lubbers, 2004; Male, 2007).

Adolescents are not only strongly influenced by their best friends, but also by their peers who have a high social status. In a series of experiments using a chat room paradigm, it has been demonstrated that adolescents increased 
their willingness to drink alcohol when popular classmates displayed pro-alcohol norms, compared with when unpopular classmates displayed the same norms (Cohen \& Prinstein, 2006; Teunissen et al., 2012). Interestingly, adolescents' social status also determines the direction of influence within best friendship dyads. Laursen, Hafen, Kerr, and Stattin (2012) examined alcohol intoxication frequency in best friendship dyads, that were distinguished based on their acceptance by their peers. Laursen and colleagues found that in stable friendships, the more accepted friends' intoxication frequency positively predicted increases in the intoxication frequency of the less accepted friends over time. In contrast, the less accepted friends' intoxication frequency did not predict the intoxication frequency of the more accepted friends over time. Similarly, Tucker, de la Haye, Kennedy, Green, and Pollard (2014) reported that adolescents were more likely to adopt the marijuana use of their more popular friends as compared with their less popular friends.

Even though the literature on similarity and influence regarding substance use in best friendship dyads is substantial, very little is known about the potential role of underlying dispositions leading to this similarity in risky behavior. Prior research has shown that adolescents can be indirectly influenced by psychological characteristics and dispositions of their best friends (Giletta, Burk, Scholte, Engels, \& Prinstein, 2013). These dispositions might exert their influence by acting as potential reinforcers of certain behaviors (e.g., friends with strong risk-taking preferences could reinforce risky behaviors, such as substance use), or by making certain norms in the peer group salient (e.g., risky behavior is cool) (Rambaran, Dijkstra, \& Stark, 2013). Additionally, adolescents might select best friends who are similar in dispositions that are associated with risky behavior, and these similarities might predate the establishment of the friendship (Lubbers, 2004; Male, 2007).

Decision-making preferences could be dispositions that lead to best friendship similarity in substance use. Adolescents' decision-making abilities are still developing, which is thought to contribute to their risky behavior, such as substance use (Boyer, 2006). Therefore, it could be hypothesized that adolescent best friends are not only similar in their substance use behavior, but also in decision-making preferences that are contributing to this behavior. Further, adolescents' decision-making might be associated with their own and their best friends' substance use. One recent study provided support for this hypothesis, by showing that adolescents reported similar risk-taking preferences as their friends, and also adjusted their risk-taking in a simulated driving task to match the risk-taking preferences of their friends who were watching and commenting on participants' driving performance (Centifanti, Modecki, MacLellan, \& Gowling, 2016). We aimed to extend these findings by: (a) including other decision-making preferences that might also contribute to similarity and influence in substance use; (b) using experimental tasks to assess decision-making preferences; and (c) exploring the role of popularity in peer influence on substance use.

Two types of decision-making preferences have been consistently linked to substance use in adolescents: risktaking preferences and the preference for immediate rewards.

Risk-taking preferences are assessed by administering gambling tasks, in which individuals make choices between risky and safe options. The risky options are usually associated with the possibility of obtaining a larger monetary reward than the safe options, but they are also associated with a higher probability of losing or not gaining money. Adolescents who display stronger risk-taking preferences in these gambling tasks relative to their peers, also report more alcohol and tobacco use (Lejuez, Aklin, Bornovalova, \& Moolchan, 2005; Xiao et al., 2009).

The preference for smaller, immediate rewards over larger, but delayed rewards is also associated with substance use (MacKillop et al., 2011). This preference is most often investigated by administering a temporal discounting (TD) task (see Scheres, de Water, \& Mies, 2013 for a review), in which individuals are asked to choose between receiving a small monetary reward (e.g., €2) today, or receiving a larger monetary reward after a delay (e.g., $€ 10$ in 30 days). The magnitude of the immediate reward and the delay preceding the delayed reward are varied, to determine the extent to which the subjective value of the delayed reward decreases with increasing delay for each individual. Adolescents who show a stronger preference for immediate rewards in TD tasks relative to their peers, also report higher levels of alcohol and tobacco use (Field, Christiansen, Cole, \& Goudie, 2007; Reynolds \& Fields, 2012).

Even though risk-taking preferences and immediate reward preferences are both positively associated with substance use, they have been proposed to be distinct constructs that are not necessarily correlated (Romer, 2010). Indeed, risk-taking preferences and immediate reward preferences are not significantly correlated in adolescents and 
young adults (de Water, Cillessen, \& Scheres, 2014; Loughran, Paternoster, \& Weiss, 2012; Olson, Hooper, Collins, \& Luciana, 2007; Scheres et al., 2006).

Distinct neural systems might underlie risk-taking preferences and immediate reward preferences. Dual-system models propose that adolescent risk-taking is caused by reduced connectivity between socio-emotional brain areas (e. g., the ventral striatum), and cognitive control regions (e.g., the prefrontal cortex) (Crone \& Dahl, 2012; Luciana, 2013). In line with these models, Steinberg and colleagues (2008) showed that the development of sensation-seeking (i.e., the willingness to take risks to attain highly stimulating experiences) mirrored the development of the socio-emotional neural system, while the development of self-control (conceptually similar to delayed reward preferences) mirrored the development of the cognitive control neural system.

Moreover, different components of impulsivity, TD and response inhibition, were found to be differentially associated with health-risk behaviors and criminal behaviors in adolescents (Nagin \& Pogarsky, 2004). Together, these findings suggest that risk-taking preferences and immediate reward preferences should be treated as separate constructs that might be differentially associated with substance use in adolescents.

Thus, the first goal of the current study was to investigate whether adolescent best friends are not only similar in their substance use behavior, but also in their decision-making preferences associated with these behaviors, such as risk-taking and immediate reward preferences. In addition, we examined whether adolescents' risk-taking and immediate reward preferences were associated with their own and their best friends' substance use. To our knowledge, these questions have not yet been explored.

Moreover, the handful of studies that have been conducted on the role of social status in peer influence effects on substance use within adolescent best friendships (Laursen et al., 2012; Tucker et al., 2014), have used measures of popularity that might be more strongly related to peer acceptance. For instance, Laursen and colleagues asked participants to name peers with whom they spend time and hang out with, while Tucker et al. used the number of received friendship nominations as a proxy for popularity. While popularity and acceptance are correlated in adolescents, this correlation is only moderate (Mayeux, Sandstrom, \& Cillessen, 2008), leading some researchers to argue that acceptance and popularity reflect different types of social status in adolescence (Cillessen \& Rose, 2005).

Popular adolescents are dominant, influential and highly visible and central in the peer group. They not only engage in pro-social behaviors, but also display negative behaviors, such as heightened levels of relational aggression and substance use (Cillessen \& Mayeux, 2004; Tucker et al., 2012). This mixed profile of behaviors is also reflected in the attitudes of peers toward popular adolescents; while popular adolescents are liked by their peers on the explicit level (i.e., based on peer nominations), they evoke avoidance reactions on the implicit level, as measured by an approach-avoidance task (Lansu, Cillessen, \& Karremans, 2012). Given that popular adolescents are dominant and visible, and engage in heightened levels of substance use themselves, they could influence the substance use of their best friends even more than accepted adolescents.

Therefore, the main aim of this study was threefold. We examined: (a) whether adolescent best friends were similar in their alcohol, tobacco, and marijuana use, and in their decision-making, which included risk-taking and immediate reward preferences; (b) whether adolescents' own decision-making was associated with their own substance use; and (c) the associations between adolescents own decision-making and their best friend's substance use, and whether the relative popularity of the friends affected these associations. Actor-partner interdependence models (APIM; Kenny, Kashy, \& Cook, 2006) were used to test these associations. These models control for the interdependence of the data within best friendship dyads, caused by the high degree of similarity between best friends. We controlled for age differences between the dyads in these analyses.

We predicted that adolescent best friends would be similar in their substance use and decision-making (Burk et al., 2012; Kiuru et al., 2010). We hypothesized that adolescents who showed stronger risk-taking preferences and a stronger immediate reward preference, would report enhanced substance use (Field et al., 2007; Lejuez et al., 2005; Reynolds \& Fields, 2012; Xiao et al., 2009). We expected that stronger risk-taking preferences and a stronger preference for immediate rewards of the most popular friends would be associated with increased substance use of the least popular friends (Cohen \& Prinstein, 2006; Laursen et al., 2012; Teunissen et al., 2012; Tucker et al., 2014). 


\section{METHOD}

\section{1 | Participants}

A total of 172 adolescents aged $12-18$ years participated in this study (48\% female, $M_{\text {age }}=15.22, \mathrm{SD}=1.51$ ). Most participants were Dutch (85.5\%), with the remaining 14.5\% from various ethnic backgrounds (e.g., Turkish, Moroccan, Surinamese, and Indonesian). Participants were drawn from eight classrooms of a high school in The Netherlands. Classrooms ranged in size from 20 to 29 students $(M=23.88, S D=3.04)$.

Prior to the study, parents of the participants were sent an information letter describing the goals and procedures of the study. If they did not want their child to participate, they were asked to notify the experimenter before the start of the study. The parents of only one of the contacted participants denied participation of their child in the current study. Moreover, absenteeism on the days of testing was relatively low, in that on average $91.4 \%$ (SD $=6.0 \%$; range $=80-100 \%$ ) of the students in each class participated in the present study. All procedures were approved by the Internal Review Board at the authors' research institute.

\section{$2.2 \mid$ Measures}

\subsection{1 | Popularity}

Participants rated the popularity of each of their classmates on a 7-point scale (ranging from $1=$ not popular at all to 7 = extremely popular). The mean of all classmates' ratings for each participant was used as a measure of popularity for each adolescent. We also asked participants to nominate classmates they thought were most popular and least popular. The popularity score computed from these peer nominations (number of most popular nominations received minus least popular nominations received, both standardized within the classroom) correlated highly with the popularity score derived from the popularity ratings $(r h o=.90$ ). Given that ratings might be more sensitive to subtle inter-individual differences, we used the popularity ratings to distinguish the dyad members on their popularity. ${ }^{1}$

\subsection{2 | Best friendships}

To determine best friendship dyads, participants were asked to name their best friend in their classroom, and they were allowed to name only one friend. Based on this information, 54 unique reciprocated best friendship dyads (i.e., two adolescents who have both named each other as their best friend) were identified. These dyads consisted of 49 same-sex dyads, and 5 mixed-sex dyads. To keep the sample as homogeneous as possible, only the same-sex dyads were included in the dyadic analyses ( $n=98$ participants in 49 dyads; 50 girls, and 48 boys). Note however, that by including all 54 dyads, the results of the dyadic analyses were unchanged. Dyads were distinguished based on their popularity, with each best friendship dyad consisting of a more popular and a less popular peer. For one dyad, the rating-based popularity score was identical for both members, but they could be distinguished based on the peer nominations, since one dyad member did have a slightly higher popularity score derived from the peer nominations. For the other participants, the difference in popularity between the dyad members ranged from 0.04 to 2.44 ( $M=0.57, S D=0.53$ ).

\subsubsection{Substance use}

The Youth Risk Behavior Survey (YRBS; Brener et al., 2002) was used to measure substance use. The YRBS has high test-retest reliability in adolescents (Brener et al., 2002). Alcohol use ( $\alpha=.87$ ) was assessed by two items: one item on the frequency of recent (past 30 days) alcohol use, and one item on the frequency of binge drinking (five or more alcoholic drinks within a couple of hours) in the past 30 days. Tobacco use ( $\alpha=.87$ ) was indexed by four items: one item on the number of days tobacco was used in the past 30 days, one item on the number of cigarettes smoked per day in the past 30 days, one item on whether participants had ever smoked cigarettes daily, and one item on the frequency of smoking on school property in the past 30 days. Marijuana use was measured by one item on the number of times marijuana was used in the past 30 days.

Skewness and kurtosis statistics indicated that alcohol use was positively skewed (Skewness $=1.13, \mathrm{SE}=.24$; Kurtosis $=.33, \mathrm{SE}=.48$ ). Applying a square root transformation to the alcohol use data reduced the skewness $(.33$, 
$\mathrm{SE}=.24)$, but not the kurtosis $(-1.32, \mathrm{SE}=.48)$. Further, given that results were the same when the transformed alcohol use scores were used compared with the raw scores, we chose to report the results with the untransformed alcohol use scores in the present paper. Tobacco use (Skewness $=2.25, \mathrm{SE}=.24$; Kurtosis $=3.56, \mathrm{SE}=.48$ ) and marijuana use (Skewness $=3.16, \mathrm{SE}=.24$; Kurtosis $=10.75, \mathrm{SE}=.48$ ) were strongly positively skewed. Even after applying a square root transformation, the skewness and kurtosis remained high for tobacco use (Skewness $=1.60, \mathrm{SE}=.24$; Kurtosis $=1.35, \mathrm{SE}=.48$ ) and marijuana use (Skewness $=2.21, \mathrm{SE}=.24$; Kurtosis $=3.67, \mathrm{SE}=.48$ ). Therefore, we used dichotomous measures of tobacco and marijuana use in the analyses ( $0=$ no past month use; $1=$ past month use).

\subsubsection{Cake gambling task}

Risk-taking preferences were assessed with an adapted version of the cake gambling (CG) Task (de Water et al., 2014), in which participants made repeated choices between a high-risk option with a $33.3 \%$ probability of obtaining a relatively large monetary reward, and a low-risk option with a $66.7 \%$ probability of obtaining a smaller monetary reward. The trial procedure of the CG task is illustrated in Figure 1A. Participants viewed a cake consisting of six wedges, which were either pink or brown in a 4:2 ratio (the majority color was counterbalanced across trials). Underneath the cake, a pink square and a brown square were presented on the left and right sides (counterbalanced across trials), each containing a stack of 50 cent coins to indicate the reward associated with each color. Participants were told to choose one of the two colors by pressing the corresponding computer key, after which the computer would randomly select one of the six wedges of the cake. If the color of the selected wedge matched the color chosen by the participant, the reward associated with that color would be gained, if not, no reward would be gained. Thus, choosing the majority color is considered a low-risk choice, while choosing the minority color is considered a high-risk choice. Gain feedback was presented by showing the stack of coins, while no gain feedback was depicted by this stack of coins with a cross through them.

The rewards associated with the high-risk and low-risk choices were varied over trials (four reward magnitudes: $€ 1$ - 4 for the low-risk options, and €2-€8 for the high-risk options). The mean percentage of high-risk choices across rewards was taken as the measure of risk-taking preference. Participants first performed 8 practice trials of the task, followed by 72 experimental trials (18 repetitions of each reward magnitude), with a total duration of approximately 7 min. The trials were presented in a random order across participants.

Risk-taking preferences were internally consistent across the four reward types used in the CG task (Cronbach's $\alpha=$.93). Consistent with prior research (van Leijenhorst, Westenberg, \& Crone, 2008, 2010), a repeated measures ANOVA revealed that participants made more high-risk decisions as the reward associated with the high-risk option increased $\left(F(3,169)=13.49, p<.001, \eta_{\mathrm{p}}{ }^{2}=.19\right)$. The CG task was shown to be externally valid, in that individuals who made more high-risk decisions in this task, also reported greater sensation-seeking in daily life (van Leijenhorst et al., 2008).

\subsection{5 | TD task}

To assess the preference for immediate rewards, a TD task was administered (see Figure 1B). In each trial of this task (see de Water et al., 2014 for a detailed description), participants were required to choose between an immediate monetary reward they would receive today, or a larger reward they would receive after a delay. Five delays were used: $2,14,30,180$, and 365 days. The delayed reward was always $€ 10$. The magnitude of the immediate reward was always smaller than the delayed reward, and was adjusted based on participants' choices (Du, Green, \& Myerson, 2002). The adjustments of the immediate reward magnitude continued until participants had made six choices at a delay. After these six choices, a new delay was introduced, and participants were again presented with six choices at this delay. The separate delays were presented in a random order across participants. The subjective value of the delayed reward at each delay was defined as the magnitude of the immediate reward on a hypothetical seventh trial (Du et al., 2002). These subjective values were subsequently used to calculate the main outcome measure: area under the curve (AUC), using a procedure described by Myerson, Green, and Warusawitharana (2001). AUC ranges from 0 (in case the immediate reward is always selected) to 1 (in case the delayed reward is always selected). Thus, smaller values indicate a stronger preference for immediate rewards, while larger values indicate a stronger preference for delayed rewards. A total of 30 trials were administered. Six practice trials were administered, at a delay that was not used in the actual task (1 day). The TD task had a total duration of approximately 5 min. 


\section{A}

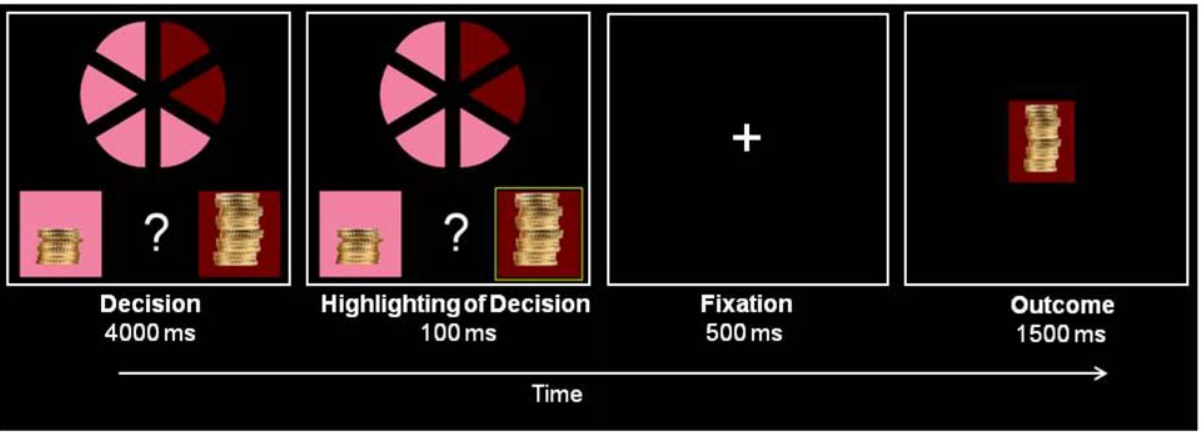

B

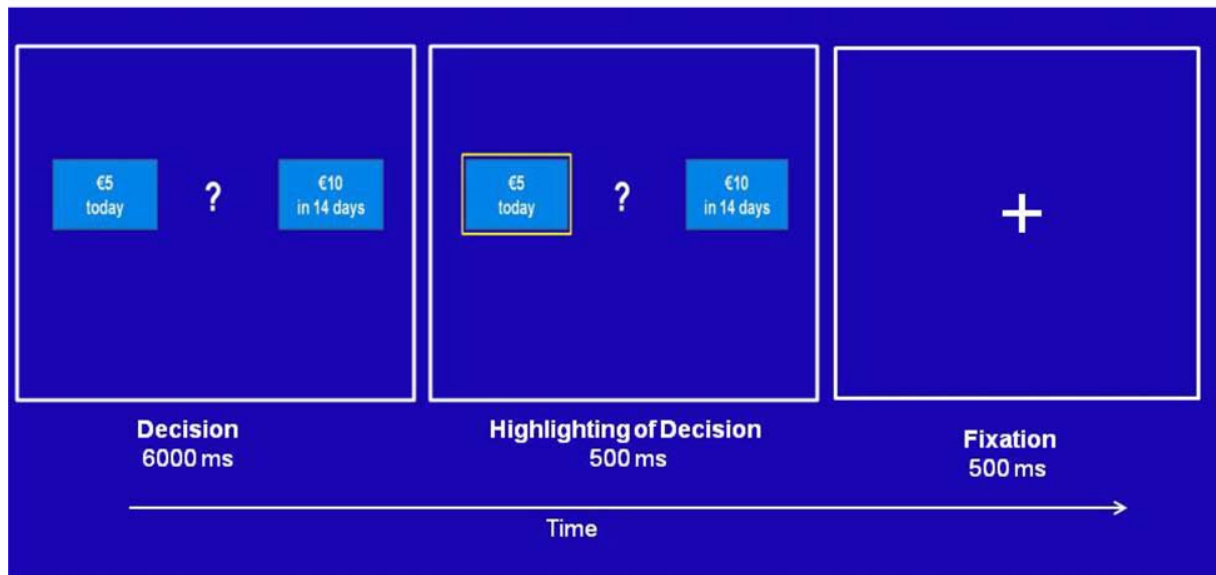

FIGURE 1 (A) Trial procedure of the cake gambling task. Participants had to chose between the pink color (low-risk choice in this trial) and brown color (high-risk choice in this trial), after which the computer randomly selected one of the six wedges of the cake. If the selected color matched the color chosen by the participant, the reward presented as a stack of 50 cent coins in the pink or brown squares underneath the cake would be gained, if not, no reward would be gained. In this trial, four could be gained by choosing the pink color, and eight could be gained by choosing the brown color. (B) Trial procedure of the temporal discounting task.

Note. The time indicated underneath the decision phase of both tasks is the maximum amount of time allowed to make a decision. When participants indicated their preference within this time period, the trial continued immediately after they pressed the button corresponding to their preference

Participants' subjective values of the delayed rewards were internally consistent across the five delays (Cronbach's $\alpha=$.87). A repeated measures ANOVA further revealed that participants showed TD: the subjective value of the delayed reward decreased significantly as the delay increased $\left(F(4,168)=78.59, p<.001, \eta_{p}{ }^{2}=.65\right)$. TD tasks have excellent test-retest reliability in adolescents ( $r$ 's up to .76 with a 2-year interval; Anokhin, Golosheykin, \& Mulligan, 2015). The TD task has high external validity as well, in that adolescents with impulse-control problems-including substance abuse, Attention Deficit Hyperactivity Disorder (ADHD) and conduct disorder-show increased preferences for immediate rewards in the TD task, compared with typically developing controls (Field et al., 2007; Patros et al., 2016; White et al., 2014).

\subsection{Procedure}

Participants were tested in their school classroom, one class at a time, using computers (notebooks). Partitions were placed at each side of the screen, to make sure that participants could not view each other's screens. A teacher was 
present, and a team of research assistants was available to answer questions of participants. At the beginning of the experiment, participants were ensured that all of their answers would remain confidential, in that their answers would not be disclosed to their teachers, parents, or classmates. Participants first completed a sociometric questionnaire (which included the best friendship question) and the popularity ratings, followed by the YRBS, the CG task, and the TD task. The experiment had a total duration of 50 min (one classroom period).

To increase motivation and ecological validity, participants were told that at the end of the CG and TD tasks, one of their outcomes of each task would be randomly selected by the computer, and that they would receive the combined outcome. In reality, to keep differences in earnings between participants minimal, their earnings were based on a random computer selection from a fixed and narrow range of rewards (€6-€9).

\section{4 | Analysis plan}

Mean differences between friends' popularity, substance use, risk-taking preference, and immediate reward preference were tested using paired-samples t-tests. Similarity between the best friends in decision-making and substance use was examined by computing Pearson correlations.

The primary analyses include a series of APIM (Kenny et al., 2006) with distinguishable dyads, estimated within a structural equation modeling framework, using Mplus version 7 (Muthén \& Muthén, 1998-2012). Dyads were distinguished based on their popularity, with each dyad consisting of a more popular and a less popular friend. The primary advantage of APIMs over conventional statistical methods (e.g., regression or ANOVA), is that the APIMs account for interdependence between dyad member's reports. This is important for this study because best friends are similar, particularly on substance use (Burk et al., 2012). Figure 2 presents a conceptual APIM. Actor effects are represented by the horizontal lines, and describe associations between adolescents' decision-making and their own substance use. Partner effects are represented by the diagonal lines and describe associations between adolescent's decision-making and their best friend's substance use. These effects are estimated in a single model, such that the partner effects are controlled for the actor effects and vice versa. The correlation between predictors (friends' decision-making preferences), and the correlation between outcomes (friends' substance use) are estimated (but not depicted in the figures) to account for similarities between friends. All measures were centered by subtracting the grand mean of all participants included in the APIM analyses, as recommended by Kenny et al. Saturated models were separately estimated for alcohol, tobacco, and marijuana use. Given that we used dichotomous variables for tobacco and marijuana use, the actor and partner effects for these variables were denoted by odds ratios. Further, to ensure adequate statistical power, separate models were estimated for risk-taking preferences, and immediate reward preferences. However, note that the results were the same when both predictors were included in a single model. The mean age of the dyads was included as predictor in all APIMs, to control for age differences between the dyads.

Chi-square difference tests were used to test whether actor or partner effects differed for the more and less popular dyad members, by individually constraining each effect to be equal across the dyad members. Significant differences between dyad members are demonstrated when the fit of the model significantly decreases after a constraint is included, as indicated by a $\chi^{2}$-statistic $\geq 3.84$ and associated $p$ value $<.05$.

There were no significant differences between participants who were included in the dyadic analyses and those who could not be matched up in reciprocated dyads, in terms of age, popularity, risk-taking preference on the gambling task, immediate reward preference on the TD task, and substance use (all $p$ 's $\geq .079$ ).

\section{3 | RESULTS}

\section{1 | Descriptive statistics}

\subsubsection{Frequency of substance use}

Substance use frequency was explored for the adolescents who were included in the dyadic analyses $(n=98)$. Past month alcohol use was reported by $58.2 \%$ of adolescents, and $35.7 \%$ engaged in binge drinking (i.e., $>5$ alcoholic 


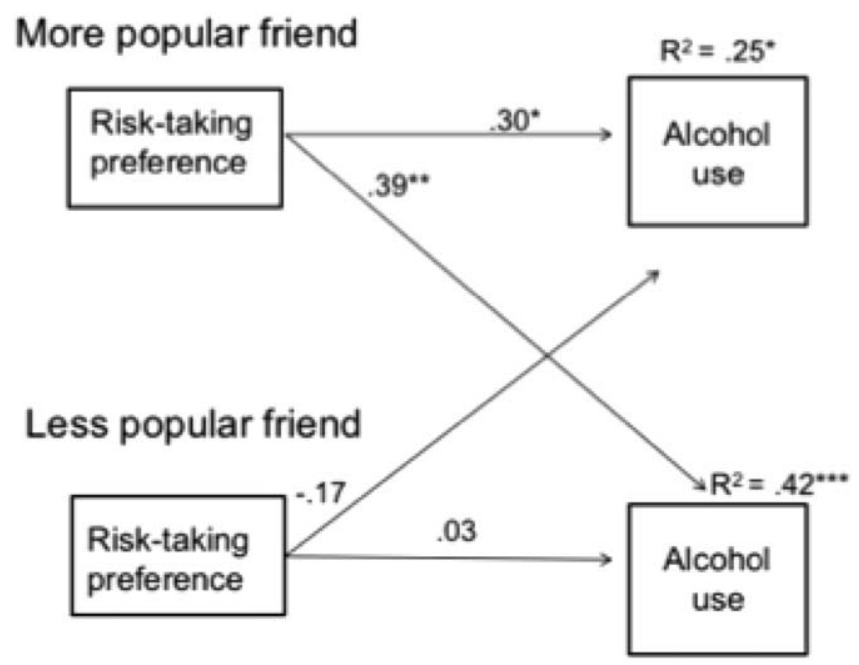

\section{More popular friend}

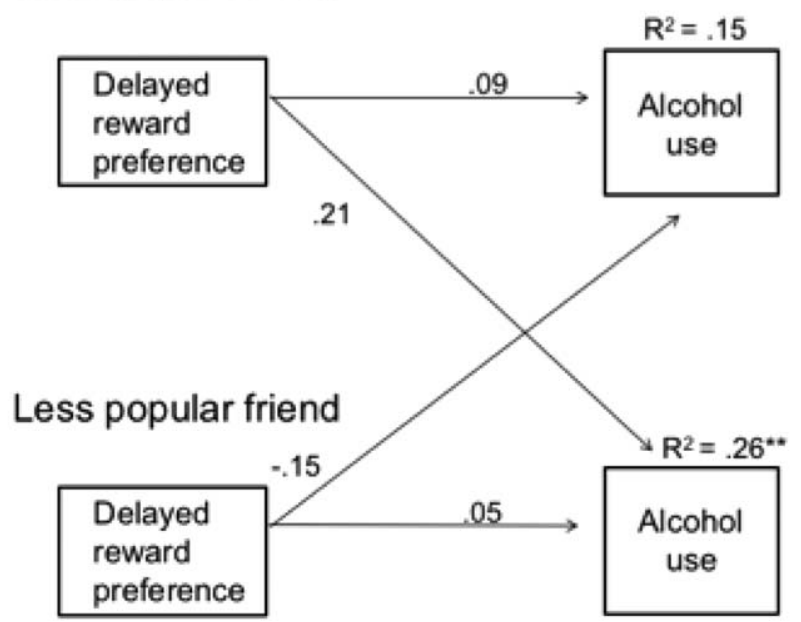

FIGURE 2 The actor-partner interdependence models for alcohol use.

Note. Standardized actor and partner effects are reported. Mean age of the dyads was included as predictor (not shown here) to control for age differences between dyads. Horizonal lines represent actor effects: the associations between adolescents' own decision-making preferences and their own alcohol use. Diagonal lines represent partner effects: the associations between adolescents' decision-making preferences and the alcohol use of their best friends. $R^{2}$ indicates the variance in alcohol use explained by the actor and partner effects. Correlations between friends' decision-making and between friends' alcohol use are estimated, but not depicted in the figure. ${ }^{*} p<.05 .{ }^{* *} p<.01 .{ }^{* * *} p<.001$

beverages within a couple of hours) in the past month. Regarding tobacco use, 18.4\% smoked cigarettes during the past month, and $10.2 \%$ indicated that they smoked cigarettes daily. Past month marijuana use was reported by $10.2 \%$ of adolescents.

\subsection{2 | Dyadic differences}

The popularity ratings of the best friends were highly correlated ( $r h o=.84)$. Nonetheless, the more popular dyad members were significantly more popular than the less popular dyad members (see Table 1). There was a trend towards more popular friends showing a stronger risk-taking preference on the gambling task $(p=.06)$ than the less popular friends. The dyad members did not differ in their age, substance use or preference for immediate rewards. 
TABLE 1 Descriptive statistics of the participants included in the APIM analyses $(n=98)$

\begin{tabular}{|c|c|c|c|c|}
\hline & Range & $\begin{array}{l}\text { More popular friend } \\
\text { M (SD) }\end{array}$ & $\begin{array}{l}\text { Less popular friend } \\
M(S D)\end{array}$ & $p$ \\
\hline Popularity rating & $1.65-6$ & $4.31(0.98)$ & $3.74(0.98)$ & $<.001$ \\
\hline Age (years) & $12.49-18.56$ & $15.44(1.48)$ & $15.34(1.45)$ & .23 \\
\hline Alcohol use & $0-5$ & $1.19(1.35)$ & $0.92(1.23)$ & .13 \\
\hline Tobacco use & $0-1$ & $0.24(0.43)$ & $0.12(0.33)$ & .06 \\
\hline Marijuana use & $0-1$ & $0.12(0.33)$ & $0.08(0.28)$ & .49 \\
\hline Risk-taking preference & $1.39-100$ & $48.52(23.34)$ & $40.37(26.14)$ & .06 \\
\hline Delayed reward preference & $.01-.99$ & $.33(.28)$ & $.32(.27)$ & .95 \\
\hline
\end{tabular}

\subsection{3 | Correlations}

In Table 2, the correlations between the study variables are shown. Popularity was positively correlated with alcohol, tobacco, and marijuana use. Age was positively correlated with alcohol use, and age was negatively correlated with the preference for immediate rewards at a trend level $(p=.054)$.

\subsection{4 | Gender differences}

Boys reported greater alcohol use than girls $(t=3.68, p<.001)$. There were no significant gender differences in tobacco use, marijuana use, risk-taking preferences, or immediate reward preferences (all p's > .16).

\subsection{Similarity in substance use and decision-making}

Best friends were similar in their alcohol use $(r=.52, p<.001)$, and tobacco use $(r=.37, p=.01)$. In addition, best friends were similar in their risk-taking preferences $(r=.31, p=.028)$, but not in their preference for immediate rewards $(r=.18, p=.227)$ or marijuana use $(r=.12, p=.427)$.

\section{3 | APIM analyses}

The results of the APIM analyses are presented in Figure 2 for alcohol use, and Figure 3 for tobacco and marijuana use. The APIMs were estimated to examine: (a) The association between adolescents own decision-making and their own substance use (actor effects); and (b) the association between adolescents own decision-making and their best friend's substance use (partner effects).

TABLE 2 Correlations between the study variables for the participants included in the APIM analyses $(n=98)$

$\begin{array}{lllll}1 . & 2 . & 3 . & 5 . & 6 .\end{array}$

1. Popularity rating

\begin{tabular}{|c|c|c|c|c|c|c|}
\hline 2. Age (years) & .11 & & & & & \\
\hline 3. Risk-taking preference & .04 & .05 & & & & \\
\hline 4. Delayed reward preference & .03 & $.20^{\dagger}$ & .11 & & & \\
\hline 5. Alcohol use & $.33^{* * *}$ & $.46^{* * *}$ & $.26^{* *}$ & .15 & & \\
\hline 6. Tobacco use & $.36^{* * *}$ & .16 & $.31^{* *}$ & -.03 & $.53^{* * *}$ & \\
\hline 7. Marijuana use & $.27^{* *}$ & .19 & $.23^{*}$ & .04 & $.52^{* * *}$ & $.71^{* * *}$ \\
\hline
\end{tabular}

Note. Tobacco and marijuana use were coded as $0=$ no use, $1=$ use.

${ }^{\dagger} p<.10 .{ }^{*} p<.05 .{ }^{* *} p<.01 .{ }^{* * *} p<.001$. 


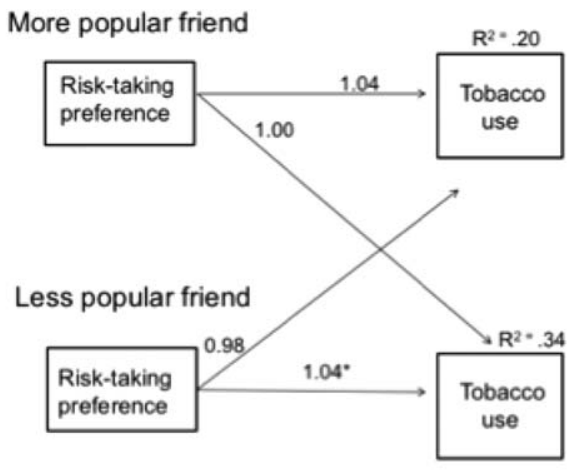

\section{More popular friend}

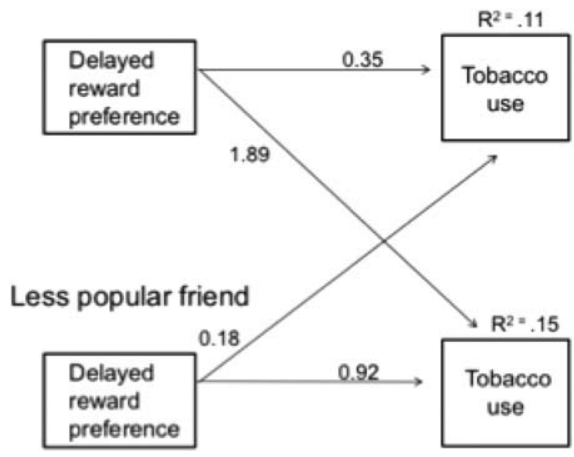

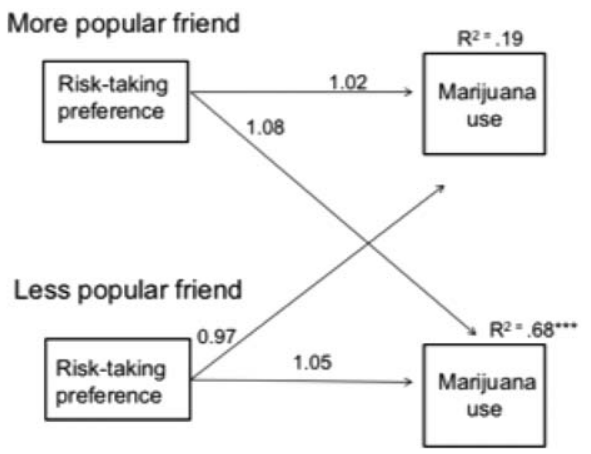

More popular friend

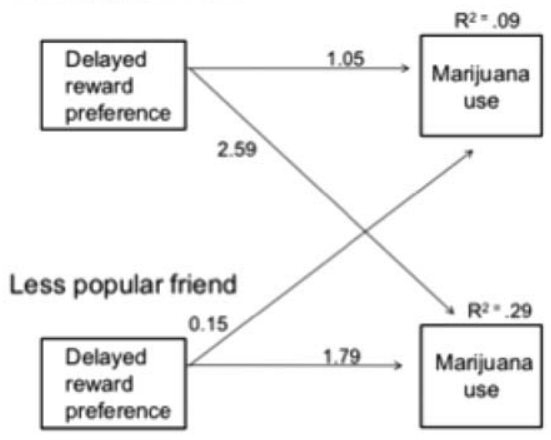

FIGURE 3 The actor-partner interdependence models for tobacco use and marijuana use.

Note. Odds ratios are reported for the actor and partner effects. Mean age of the dyads was included as predictor (not shown here) to control for age differences between dyads. Horizonal lines represent actor effects: the associations between adolescents' own decision-making preferences and their own tobacco and marijuana use. Diagonal lines represent partner effects: the associations between adolescents' decision-making preferences and the tobacco and marijuana use of their best friends. $R^{2}$ indicates the variance in tobacco or marijuana use explained by the actor and partner effects. Correlations between friends' decision-making and between friends' tobacco use and marijuana use are estimated, but not depicted in the figure. ${ }^{*} p<.05 .{ }^{* *} p<.01 .{ }^{* * *} p<.001$

\subsection{1 | Actor effects}

For the more popular friends, stronger risk-taking preferences were associated with more alcohol use $(p=.025)$. For the less popular friends, stronger risk-taking preferences were also associated with a greater likelihood of tobacco use $(p<.05)$, but not with alcohol use $(p=.791)$. There were no other significant actor effects for tobacco or marijuana use (all $p$ 's $>$.10).

\subsection{2 | Partner effects}

Stronger risk-taking preferences of the more popular friends were associated with greater alcohol use of the less popular friends $(p=.002)$. Further, this partner effect was significantly stronger for more popular friends relative to less popular friends $\left(\chi^{2}(1)=8.50, p=.003\right.$ ). There were no partner effects for tobacco and marijuana use (all $p$ 's $>.10$ ).

\subsection{3 | Controlling for popularity}

We performed additional analyses that included the average popularity scores of both dyad members to account for differences in popularity between dyads, and the absolute difference in popularity scores between dyad members to account for differences in popularity within dyads. When these two additional predictors were included, the observed actor and partner effects for alcohol use were similar. Specifically, stronger risk-taking preferences of the more popular friends remained associated with higher levels of their own alcohol use, albeit at a trend level $(\beta=.25, p=.059)$, and 
with higher levels of their less popular friends' alcohol use $(\beta=.35, p=.003)$. This means that the observed actor and partner effects do not differ for dyads in which the difference in popularity between the dyad members is large, compared with dyads in which the difference in popularity is small.

\section{4 | DISCUSSION}

The main aim of the current study was threefold. APIM analyses were performed to examine: (a) whether adolescent best friendship dyads are similar in their substance use and decision-making, which included risk-taking and immediate reward preferences; (b) whether adolescents' decision-making is associated with their own substance use (actor effects); and (c) whether adolescents' decision-making is associated with their best friend's substance use (partner effects). Further, we investigated whether the relative popularity of the friends influenced these associations.

\subsection{Similarity in substance use and decision-making}

Consistent with our predictions and with previous findings (Burk et al., 2012; Kiuru et al., 2010), best friends were similar in their alcohol and tobacco use. In addition, best friends were similar in their risk-taking preferences on a gambling task. This finding is in line with the findings of Centifanti and colleagues (2016), who showed that adolescent friends are similar in their self-reported risk-taking preferences. To our knowledge, we are the first to demonstrate that adolescent best friends are not only similar in their substance use behavior, but also in an experimentally measured (i.e., by a gambling task) decision-making preference that is thought to underpin this behavior.

Two processes have been proposed to account for this similarity within best friendship dyads: selection and socialization (Lubbers, 2004; Male, 2007). Selection takes place prior to the establishment of the friendship, and refers to choosing a friend based on similarities in certain attributes, such as risk-taking preferences (Lubbers, 2004; Male, 2007). Socialization occurs when the friendship has already been established, and refers to friends becoming more similar to each other over time, because they influence each other. For instance, adolescents with relatively strong risktaking preferences could encourage the alcohol and tobacco use of their best friend, which would make the friends more similar in their substance use over time (cf. Centifanti et al., 2016).

We found that adolescent best friends were similar in their risk-taking preference, but not in their immediate reward preference. This supports the notion that risk-taking and the preference for immediate rewards are distinct constructs (de Water et al. 2014; Loughran et al., 2012; Olson et al., 2007; Scheres et al., 2006), which may be differentially related to health-risk behaviors (Nagin \& Pogarsky, 2004), and which might have distinct underlying neural systems (Steinberg, 2008). The preference for immediate rewards is highly stable, which has led some researchers to argue that it is akin to a personality trait (Odum, 2011). Therefore, an individual's preference for immediate rewards may be less likely to be impacted by socialization processes compared to an individual's risk-taking preference, which could explain the lack of best friendship similarity in immediate reward preferences.

\subsection{Decision-making and own substance use (actor effects)}

In line with prior studies (Lejuez et al., 2005; Xiao et al., 2009), we found that adolescents who showed stronger risktaking preferences on the gambling task also reported higher levels of alcohol use, and a greater likelihood of past month tobacco use. This association was only significant for the more popular friends regarding alcohol use, and for the less popular friends regarding tobacco use.

Contrary to the risk-taking preference findings and previous research (Field et al., 2007; Reynolds \& Fields, 2012), the preference for immediate rewards on the TD task was not associated with substance use in the current study. Nevertheless, this finding does fit with a study by Nagin and Pogarsky (2004), who reported that different components of impulsivity are differentially associated with health-risk behaviors. It might be argued that TD tasks are particularly suited for distinguishing individuals at extreme ends of the impulsivity spectrum: those who are highly impulsive from those with excellent self-control. Indeed, Field et al. found that adolescents who consumed 23 alcoholic drinks 
per week on average, showed a stronger preference for immediate rewards than adolescents who consumed 3 alcoholic drinks per week on average. Similarly, Reynolds and colleagues observed that adolescents who smoked at least one cigarette a day during the last 3 months, showed a stronger preference for immediate rewards relative to adolescents who had never smoked. The levels of substance use in this study might not have been extreme enough to find an association between TD and substance use. For instance, only $10 \%$ of the participants in our study reported to have smoked cigarettes daily.

\subsection{Decision-making and best friend's substance use (partner effects)}

In keeping with our hypotheses, we found that the most popular friends' risk-taking preferences were positively associated with the alcohol use of their less popular friend. The risk-taking and immediate reward preferences of the least popular friends were not associated with the alcohol use of their more popular friends.

The finding that the relative popularity of best friends influences the association between adolescents' own risktaking preferences and their best friends' alcohol use, is consistent with chat room studies which demonstrated that popular adolescents exert a greater influence on the risky choices of their peers than unpopular adolescents (Cohen \& Prinstein, 2006; Teunissen et al., 2012). This finding is also consistent with the results of Laursen et al. (2012), who showed that the intoxication frequency of the more accepted friend within an adolescent best friendship dyad positively predicted the intoxication frequency of the less accepted friend, while the reverse association was not significant.

There are several potential explanations for the positive association between the risk-taking preferences of the more popular friends and the alcohol use of the less popular friends. First, the more popular friends might actively influence their less popular friends (cf. Centifanti et al., 2016). One form of active peer influence is deviancy training (Dishion, Capaldi, Spracklen, \& Li, 1995), in which friends influence each other by positively reinforcing (either verbally or nonverbally) each other's anti-social behavior, including substance use. Additionally, the more popular friends might bring their less popular friends into environments where there are opportunities to engage in alcohol use, such as parties. Since the more popular friends also showed a stronger risk-taking preference than their less popular friends (at a trend level) in this study, they might influence the behavior of their best friend passively as well. According to social learning theory (Bandura, 1977), people learn by imitating, or modeling, the behavior of others. It could be that the less popular friends in our study copied the alcohol use of their more popular friends, who served as potent models of this risky behavior.

Contrary to the findings for alcohol use, there were no partner effects for tobacco and marijuana use. In other words, the decision-making of the most popular friends was not associated with the tobacco and marijuana use of the least popular friends. Different motivations for using each substance might contribute to these discrepant findings across substances. Social motivations (e.g., making parties more enjoyable) are the most important reasons for adolescents to engage in alcohol use (Kuntsche, Knibbe, Gmel, \& Engels, 2005), while enhanced tobacco use is often indicative of the addictive nature of cigarettes (Kandel, Chen, Warner, Kessler, \& Grant, 1997), and experimentation is the primary motive to engage in marijuana use (Lee, Neighbors, \& Woods, 2007). The fact that social motivations are linked so strongly to alcohol use, might explain why partner effects were only significant for alcohol use in the present investigation. Alternatively, the lack of partner effects for tobacco and marijuana use could be due to the limited variance in these variables, which might have made it difficult to find an effect. Indeed, Tucker et al. (2014) did find that adolescents were more likely to adopt the marijuana use of their friends, but in their sample past month marijuana use was markedly higher (up to $29.9 \%$ of adolescents) compared to our sample (10.2\% of adolescents).

Finally, partner effects were only observed for risk-taking preferences, and not for immediate reward preferences. This is consistent with the notion that risk-taking and the preference for immediate rewards are distinct constructs (de Water et al., 2014; Loughran et al., 2012; Olson et al., 2007; Scheres et al., 2006), which may be differentially related to health-risk behaviors (Nagin \& Pogarsky, 2004), and which might have distinct underlying neural systems (Steinberg, 2008). In this study, only risk-taking preferences were associated with substance use, which may reflect the fact that 
TD tasks are particularly sensitive to extreme levels of impulsivity, and the substance use in our study was relatively moderate.

\subsection{Strengths and limitations}

To the best of our knowledge, our study is the first to combine experimental decision-making tasks with measures of popularity and best friendship derived from sociometric questionnaires. By including these experimental tasks, we were able to provide more detailed insights into similarities and influence regarding substance use within best friendship dyads. Furthermore, the use of APIMs allowed us to study how the decision-making of adolescents was related to the substance use of their best friend, while controlling for similarity in both decision-making and substance use. Our finding that the risky decision-making of the more popular friends was associated with the substance use of the less popular friends has important implications for substance abuse preventions and interventions. Risky decision-making can be reduced in adolescents by cognitive interventions (Reyna, Weldon, \& McCormick, 2015), and these interventions could be focused particularly on more popular adolescents.

Nonetheless, several limitations of the current study need to be mentioned. The number of dyads in the present study $(n=49)$ was relatively modest. This number is larger than the 36 dyads needed to test for dyadic similarity with a power $\geq .80$ (Kenny, Kashy, \& Bolger, 1998). Nevertheless, the relatively modest number of dyads limits the generalizability of our findings. Future studies need to replicate our findings in larger samples, which would also allow for the examination of additional moderators, such as gender effects. Due to the cross-sectional design of our study, the direction of the associations between the decision-making of the most popular friend and the alcohol use of the least popular friend could not be determined. Future studies should examine these associations using a longitudinal design, to determine whether increases in the most popular friends' risk-taking preferences over time are also associated with increases in the least popular friends' alcohol use. Additionally, longitudinal designs would enable researchers to test whether the associations between decision-making and alcohol use are bidirectional, since alcohol use might also affect decision-making. Further, our proposed explanations for the observed partner effects (e.g., active peer influence, modeling) need to be tested in observational or experimental studies. Additionally, we did not assess the duration and quality of the friendships, which could have influenced the strength of the associations. Given that friends in reciprocated friendships are more similar than friends in nonreciprocated friendships (Tucker et al., 2014), it might be argued that friends who report a higher friendship quality or duration are also more similar and influence each other more strongly. This interesting possibility needs to be addressed in future investigations.

Finally, future studies should extend our findings by using different decision-making tasks (e.g., a simulated driving task or a TD task in which all rewards and delays are experienced), to better understand what these tasks are tapping.

\section{5 | Conclusions}

We found that adolescent best friends are similar in their alcohol use, tobacco use, and in their risk-taking preferences on a gambling task. The risk-taking preferences of the most popular friends were associated with higher levels of alcohol use of the least popular friends. These findings indicate that adolescent best friends are similar in their substance use behavior and their risky decision-making, and underscore the influence popular adolescents have on the substance use of their peers, including their best friends. These findings may have practical implications as well. Interventions aimed at preventing alcohol abuse in adolescents might benefit from focusing specifically on decreasing the risky decision-making of the more popular friends, or on increasing the resistance to peer influence of the less popular friends. For instance, cognitive training programs have been shown to reduce risky decision-making in adolescents (Reyna et al., 2015). These programs might be administered in combination with resistance to peer influence training, to evaluate whether they reduce risky decision-making and the associated substance use in adolescents. 


\section{ACKNOWLEDGEMENTS}

The authors would like to thank Linda van Leijenhorst for providing them with the Cake Gambling Task. We are grateful to Giovanni ten Brink for programming computerized versions of the questionnaires that we used in the present study. We would like to acknowledge Nan Stevens for translating the Youth Risk Behavior Survey. For their assistance during data collection, the authors would like to thank: Lena Gleumes, Ilse de Rijk, Fleur Robben, Maartje Verhees, and Maurice te Wierik. Finally, we are indebted to all participating adolescents and teachers. This research was financed by VIDI grant 452-09-004 awarded to Anouk Scheres by the Netherlands Organisation for Scientific Research (NWO). The funding agency was not involved in the study design, collection, analysis or interpretation of the data, nor in the writing of the report, or the decision to submit the article for publication.

\section{NOTE}

${ }^{1}$ Note that when the popularity score derived from the peer nominations was used to distinguish the dyad members on their popularity, six dyads (12.2\%) were distinguished differently compared with the ratings-based popularity score. However, when we distinguished the dyads based on the popularity score derived from the peer nominations, and repeated the APIM analyses, the findings were similar to the reported findings.

\section{REFERENCES}

Anokhin, A. P., Golosheykin, S., \& Mulligan, R. C. (2015). Long-term test-retest reliability of delayed reward discounting in adolescents. Behavioural Processes, 111, 55-59. doi: 10.1016/j.beproc.2014.11.008

Bandura, A. (1977). Social learning theory. New York: General Learning Press.

Boyer, T. W. (2006). The development of risk-taking: A multi-perspective review. Developmental Review, 26, 291-345. doi: 10.1016/j.dr.2006.05.002

Brener, N. D., Kann, L., McManus, T., Kinchen, S. A., Sundberg, E. C., \& Ross, J. G. (2002). Reliability of the 1999 Youth Risk Behavior Survey questionnaire. Journal of Adolescent Health, 31, 336-342. doi: 10.1016/s1054-139x(02)00339-7

Burk, W. J., van der Vorst, H., Kerr, M., \& Stattin, H. (2012). Alcohol use and friendship dynamics: Selection and socialization in early-, middle-, and late-adolescent peer networks. Journal of Studies on Alcohol and Drugs, 73, 89-98.

Centifanti, L. C. M., Modecki, K. L., MacLellan, S., \& Gowling, H. (2016). Driving under the influence of risky peers: An experimental study of adolescent risk taking. Journal of Research on Adolescence, 26, 207-222. doi: 10.1111/ jora.12187

Cillessen, A. H. N., \& Mayeux, L. (2004). From censure to reinforcement: Developmental changes in the association between aggression and social status. Child Development, 75, 147-163. doi: 10.1111/j.1467-8624.2004.00660.x

Cillessen, A. H. N., \& Rose, A. J. (2005). Understanding popularity in the peer system. Current Directions in Psychological Science, 14, 102-105. doi: 10.1111/j.0963-7214.2005.00343.x

Cohen, G. L., \& Prinstein, M. J. (2006). Peer contagion of aggression and health risk behavior among adolescent males: An experimental investigation of effects on public conduct and private attitudes. Child Development, 77, 967-983. doi: 10.1111/j.1467-8624.2006.00913.x

Crone, E. A., \& Dahl, R. E. (2012). Understanding adolescence as a period of social-affective engagement and goal flexibility. Nature Reviews Neuroscience, 13, 636-650. doi:10.1038/nrn3313

de Water, E., Cillessen, A. H. N., \& Scheres, A. (2014). Distinct age-related differences in temporal discounting and risk taking in adolescents and young adults. Child Development, 85, 1881-1897. doi: 10.1111/cdev.12245

Dishion, T. J., Capaldi, D., Spracklen, K. M., \& Li, F. Z. (1995). Peer ecology of male-adolescent drug-use. Development and Psychopathology, 7, 803-824.

Du, W. J., Green, L., \& Myerson, J. (2002). Cross-cultural comparisons of discounting delayed and probabilistic rewards. Psychological Record, 52, 479-492.

Field, M., Christiansen, P., Cole, J., \& Goudie, A. (2007). Delay discounting and the alcohol Stroop in heavy drinking adolescents. Addiction, 102, 579-586. doi: 10.1111/j.1360-0443.2007.01743.x

Gardner, M., \& Steinberg, L. (2005). Peer influence on risk taking, risk preference, and risky decision making in adolescence and adulthood: An experimental study. Developmental Psychology, 41, 625-635. doi: 10.1037/00121649.41.4.625

Giletta, M., Burk, W. J., Scholte, R. H. J., Engels, R., \& Prinstein, M. J. (2013). Direct and indirect peer socialization of adolescent nonsuicidal self-injury. Journal of Research on Adolescence, 23, 450-463. doi: 10.1111/jora.12036 
Kandel, D., Chen, K., Warner, L. A., Kessler, R. C., \& Grant, B. (1997). Prevalence and demographic correlates of symptoms of last year dependence on alcohol, nicotine, marijuana and cocaine in the US population. [Article]. Drug and Alcohol Dependence, 44, 11-29. doi: 10.1016/s0376-8716(96)01315-4

Kenny, D. A., Kashy, D., \& Bolger, N. (1998). Data analysis in social psychology. In D. Gilbert, S. Fiske \& G. Lindzey (Eds.), Handbook of social psychology (4th ed., pp. 233-265). New York: McGraw-Hill.

Kenny, D. A., Kashy, D. A., \& Cook, W. L. (2006). Analysis of dyadic data. New York: Guilford.

Kiuru, N., Burk, W. J., Laursen, B., Salmela-Aro, K., \& Nurmi, J. E. (2010). Pressure to drink but not to smoke: Disentangling selection and socialization in adolescent peer networks and peer groups. Journal of Adolescence, 33, 801-812. doi: 10.1016/j.adolescence.2010.07.006

Kuntsche, E., Knibbe, R., Gmel, G., \& Engels, R. (2005). Why do young people drink? A review of drinking motives. Clinical Psychology Review, 25, 841-861. doi: 10.1016/j.cpr.2005.06.002

LaFontana, K. M., \& Cillessen, A. H. N. (2010). Developmental changes in the priority of perceived status in childhood and adolescence. Social Development, 19, 130-147. doi: 10.1111/j.1467-9507.2008.00522.x

Lansu, T. A. M., Cillessen, A. H. N., \& Karremans, J. C. (2012). Implicit associations with popularity in early adolescence: An approach-avoidance analysis. Developmental Psychology, 48, 65-75. doi: 10.1037/a0025681

Laursen, B., Hafen, C. A., Kerr, M., \& Stattin, H. (2012). Friend influence over adolescent problem behaviors as a function of relative peer acceptance: To be liked is to be emulated. Journal of Abnormal Psychology, 121, 88-94. doi: 10.1037/a0024707

Lee, C. M., Neighbors, C., \& Woods, B. A. (2007). Marijuana motives: Young adults' reasons for using marijuana. Addictive Behaviors, 32, 1384-1394. doi: 10.1016/j.addbeh.2006.09.010

Lejuez, C. W., Aklin, W., Bornovalova, M., \& Moolchan, E. T. (2005). Differences in risk-taking propensity across inner-city adolescent ever- and never-smokers. Nicotine and Tobacco Research, 7, 71-79. doi: 10.1080/14622200412331328484

Loughran, T. A., Paternoster, R., \& Weiss, D. (2012). Hyperbolic time discounting, offender time preferences and deterrence. Journal of Quantitative Criminology, 28, 607-628. doi: 10.1007/s10940-011-9163-5

Lubbers, M. J. (2004). The social fabric of the classroom. Peer relations in secundary education. Veenendaal: Universal Press.

Luciana, M. (2013). Adolescent brain development in normality and psychopathology. Development and Psychopathology, 25, 1325-1345. doi: 10.1017/s0954579413000643

MacKillop, J., Amlung, M. T., Few, L. R., Ray, L. A., Sweet, L. H., \& Munafo, M. R. (2011). Delayed reward discounting and addictive behavior: A meta-analysis. Psychopharmacology, 216, 305-321. doi: 10.1007/s00213-011-2229-0

Male, D. B. (2007). Friendship and peer relationships. In L. Florian (Ed.), The SAGE handbook of special education (pp. 460471). London: SAGE.

Mayeux, L., Sandstrom, M. J., \& Cillessen, A. H. N. (2008). Is being popular a risky proposition?. Journal of Research on Adolescence, 18, 49-74. doi: 10.1111/j.1532-7795.2008.00550.x

Muthén, L. K., \& Muthén, B. O. (1998-2012). Mplus user's guide (7th ed.). Los Angeles, CA: Muthén \& Muthén.

Myerson, J., Green, L., \& Warusawitharana, M. (2001). Area under the curve as a measure of discounting. Journal of the Experimental Analysis of Behavior, 76, 235-243. doi: 10.1901/jeab.2001.76-235

Nagin, D. S., \& Pogarsky, G. (2004). Time and punishment: Delayed consequences and criminal behavior. Journal of Quantitative Criminology, 20, 295-317. doi: 10.1007/s10940-004-5866-1

Odum, A. L. (2011). Delay discounting: Trait variable?. Behavioural Processes, 87, 1-9. doi: 10.1016/j.beproc.2011.02.007

Olson, E. A., Hooper, C. J., Collins, P., \& Luciana, M. (2007). Adolescents' performance on delay and probability discounting tasks: Contributions of age, intelligence, executive functioning, and self-reported externalizing behavior. Personality and Individual Differences, 43, 1886-1897. doi: 10.1016/j.paid.2007.06.016

Patros, C. H., Alderson, R. M., Kasper, L. J., Tarle, S. J., Lea, S. E., \& Hudec, K. L. (2016). Choice-impulsivity in children and adolescents with attention-deficit/hyperactivity disorder (ADHD): A meta-analytic review. Clinical Psychology Review, 43, 162-174. doi: 10.1016/j.cpr.2015.11.001

Rambaran, A. J., Dijkstra, J. K., \& Stark, T. H. (2013). Status-based influence processes: The role of norm salience in contagion of adolescent risk attitudes. Journal of Research on Adolescence, 23, 574-585. doi: 10.1111/jora.12032

Reyna, V. F., Weldon, R. B., \& McCormick, M. (2015). Educating intuition: Reducing risky decisions using fuzzy-trace theory. Current Directions in Psychological Science, 24, 392-398. doi: 10.1177/0963721415588081

Reynolds, B., \& Fields, S. (2012). Delay discounting by adolescents experimenting with cigarette smoking. Addiction, 107, 417-424. doi: 10.1111/j.1360-0443.2011.03644.x

Romer, D. (2010). Adolescent risk taking, impulsivity, and brain development: Implications for prevention. Developmental Psychobiology, 52, 263-276. doi: 10.1002/dev.20442

Scheres, A., de Water, E., \& Mies, G. W. (2013). The neural correlates of temporal reward discounting. WIREs Cognitive Science, 4, 523-545. doi: 10.1002/wcs.1246 
Scheres, A., Dijkstra, M., Ainslie, E., Balkan, J., Reynolds, B., Sonuga-Barke, E., \& Castellanos, F. X. (2006). Temporal and probabilistic discounting of rewards in children and adolescents: Effects of age and ADHD symptoms. Neuropsychologia, 44, 2092-2103. doi: 10.1016/j.neuropsychologia.2005.10.012

Steinberg, L. (2008). A social neuroscience perspective on adolescent risk-taking. Developmental Review, 28, 78-106. doi: 10.1016/j.dr.2007.08.002

Steinberg, L., Albert, D., Cauffman, E., Banich, M., Graham, S., \& Woolard, J. (2008). Age differences in sensation seeking and impulsivity as indexed by behavior and self-report: Evidence for a dual systems model. Developmental Psychology, 44, 1764-1778. doi: 10.1037/a0012955

Steinberg, L., \& Morris, A. S. (2001). Adolescent development. Annual Review of Psychology, 52, 83-110. doi: 10.1146/ annurev.psych.52.1.83

Teunissen, H. A., Spijkerman, R., Prinstein, M. J., Cohen, G. L., Engels, R. C., \& Scholte, R. H. J. (2012). Adolescents' conformity to their peers' pro-alcohol and anti-alcohol norms: The power of popularity. Alcoholism-Clinical and Experimental Research, 36, 1257-1267. doi: 10.1111/j.1530-0277.2011.01728.x

Tucker, J. S., de la Haye, K., Kennedy, D. P., Green, H. D., \& Pollard, M. S. (2014). Peer influence on marijuana use in different types of friendships. Journal of Adolescent Health, 54, 67-73. doi: 10.1016/j.jadohealth.2013.07.025

Tucker, J. S., Miles, J. N. V., D'amico, E. J., Zhou, A. J., Green, H. D., \& Shih, R. A. (2012). Is popularity hazardous to adolescents' health?: Cross-sectional and longitudinal associations with adolescent substance use. Alcoholism-Clinical and Experimental Research, 36, 327A-327A.

Van Leijenhorst, L., Gunther Moor, B., de Macks, Z. A. O., Rombouts, S. A. R. B., Westenberg, P. M., \& Crone, E. A. (2010). Adolescent risky decision-making: Neurocognitive development of reward and control regions. Neuroimage, 51, 345-355. doi: 10.1016/j.neuroimage.2010.02.038

Van Leijenhorst, L., Westenberg, P. M., \& Crone, E. A. (2008). A developmental study of risky decisions on the cake gambling task: Age and gender analyses of probability estimation and reward evaluation. Developmental Neuropsychology, 33, 179-196. doi: 10.1080/87565640701884287

White, S. F., Clanton, R., Brislin, S. J., Meffert, H., Hwang, S., Sinclair, S., \& Blair, R. J. R. (2014). Temporal discounting and conduct disorder in adolescents. Journal of Personality Disorders, 28, 5-18.

Xiao, L., Bechara, A., Grenard, J., Stacy, W. A., Palmer, P., Wei, Y. L., .. Johnson, C. A. (2009). Affective decision-making predictive of Chinese adolescent drinking behaviors. Journal of the International Neuropsychological Society, 15, 547557. doi: $10.1017 / \mathrm{s} 1355617709090808$

How to cite this article: de Water E, Burk WJ, Cillessen AHN, Scheres A. Substance use and decision-making in adolescent best friendship dyads: The role of popularity. Social Development. 2017;26:860-875. https://doi. org/10.1111/sode.12227 